\title{
Rationale for switching to nonlocal functionals in Density Functional Theory
}

\author{
P Lazić ${ }^{1}, \mathbf{N}$ Atodiresei $^{2}$, V Caciuc $^{2}$, R Brako ${ }^{3}$, B Gumhalter ${ }^{4}$ \\ and $\mathrm{S}$ Blügel ${ }^{2}$ \\ ${ }^{1}$ Department of Materials Science and Engineering, Massachusetts Institute of \\ Technology, Cambridge, Massachusetts 02139 \\ ${ }^{2}$ Institut für Festkörperforschung (IFF) and Institute for Advanced Simulation \\ (IAS), Forschungszentrum Jülich, 52425 Jülich, Germany \\ ${ }^{3}$ Rudjer Bošković Institute, P.O. Box 180, Bijenička c. 54, 10002 Zagreb, Croatia \\ ${ }^{4}$ Institute of Physics, Bijenička c. 46, 10002 Zagreb, Croatia \\ E-mail: plazic@mit.edu
}

\begin{abstract}
The Density Functional Theory (DFT) has been steadily improving over the past few decades, becoming the standard tool for electronic structure calculations. The early local functionals (LDA) were eventually replaced by more accurate semi-local functionals (GGA) which are in use today. A major persisting drawback is the lack of the nonlocal correlation which is at the core of dispersive (van der Waals) forces, so that a large and important class of systems remains outside the scope of DFT. The vdW-DF correlation functional of Langreth and Lundqvist published in 2004 was the first nonlocal functional which could be easily implemented. Beyond expectations, the nonlocal functional have brought significant improvement to systems that were believed not to be sensitive to nonlocal correlations. In this paper we use the example of graphene nanodomes growing on $\operatorname{Ir}(111)$ surface, where with the increase of the size of the graphene islands the character of the bonding changes from strong chemisorption towards almost pure physisorption. We demonstrate how the seamless character of the vdW-DF functionals makes it possible to treat all regimes selfconsistently, proving to be a systematic and consistent improvement of DFT regardless of the nature of bonding. We also discuss the typical surface science example of CO adsorption on (111) surfaces of metals, which shows that the nonlocal correlation may also be crucial for strongly chemisorbed systems. We briefly discuss open questions, in particular the choice of the most appropriate exchange part of the functional. As the vdW-DF begins to appear implemented selfconsistently in a number of popular DFT codes, with numerical cost close to the GGA calculations, we draw the attention of the DFT community to the advantages and benefits of adoption of this new class of functionals.
\end{abstract}

PACS numbers: 68.65.-k, 68.43-h, 71.15.Mb 


\section{Introduction}

Several years have passed since the introduction of the first practical nonlocal density functional vdW-DF in 2004 [1, 2], but the local and semi-local functionals still continue to dominate the density functional theory [3] (DFT) calculations. One reason for the slow acceptance of vdW-DF was the unavailability of codes that implement the nonlocal functional. The implementations were usually not publicly available, they were numerically demanding and not selfconsistent, with few exceptions like the postprocessing implementation JuNoLo [4] which can be applied to the results of most standard DFT codes. This has changed recently, as self-consistent implementations based on the efficient algorithm of Román-Pérez and Soler [5] appeared in several standard programs. Another reason of the slow progress towards nonlocal functionals has been the belief that standard semilocal density functionals are satisfactory in the case of predominantly chemically bonded systems, and that in cases of predominantly physisorbed systems the results could be amended by recently developed semiempirical schemes - such as DFT-D $[6,7,8]$ - that include the van der Waals (vdW) interaction. In this paper we show that these approaches also have problems and limitations, which can be systematically resolved using nonlocal functionals.

With the very efficient implementation of the vdW-DF functional [5] using the interpolation which enables the use of fast Fourier transform and reduces the calculation complexity down to $N \log N$ instead of $N^{2}$, where $N$ is a number of charge density grid points, the cost of selfconsistent calculations becomes virtually the same as for the semilocal GGA functional. The implementation of this method has recently been incorporated in several standard programs including GPAW [9] and the most recent versions of VASP $[10,11,12]$. Besides the great advantage of having only the logarithmic complexity, this implementation has some disadvantages [13], one of them being that nonlocal correlation binding energy can not be obtained in real space. Visualisation of this quantity may elucidate the binding mechanism, and we have therefore used the postprocessing approach with JuNoLo code [4] in which this can be carried out.

To demonstrate the capabilities of nonlocal functionals we have examined a system in which the interaction covers the range from strong chemisorption regime to pure vdW binding without a change in chemical composition, i.e. just by changing its geometrical structure. The system chosen is the epitaxial growth of graphene on $\operatorname{Ir}(111)$ surface, in which the initial strong bonds of newly added carbon atoms at the edges of the structure make way to much weaker vdW interactions inside the growing graphene flake. Since the system makes a continuous transition from chemisorption to physisorption the only way to calculate it in DFT is to have a seamless functional that covers all adsorption regimes. Even though the vdW-DF was initially developed to bring sparse matter systems (in which vdW interactions dominate) within the grasp of DFT [14], the method was derived from the adiabatic-connection fluctuation-dissipation theorem (ACFDT) in a seamless fashion, in order to retain correct description regardless of the dominant nature of bonding in the studied system. This seamless quality was not stressed enough 
in the early papers and the choice of the name of the functional may have obscured its potential.

Starting from the ACFDT there are two possible directions of development. One was taken by Dion et al. [1] in deriving vdW-DF, attempting to obtain the response function which enables the construction of the true density functional in the spirit of the DFT, requiring only the charge density to calculate the total energy. The other one proceeds in the direction of many body perturbation theory and towards the random phase approximation (RPA) [15]. Starting from the same origin and having the same goal these two approaches should produce equal results regarding the total energy, with the additional advantage of the vdW-DF being computationally much cheaper than the RPA, as was already recognized in [16]. The fact that vdW-DF deals only with correlation leaves the question of the best accompanying exchange part of the functional open. The problem of the appropriate exchange functional has been addressed recently on several occasions, including the revised version of the vdW-DF $[17,18,19]$.

In our analysis of the performance of nonlocal functionals we first present the calculations of the growth of graphene on $\operatorname{Ir}(111)$, and then turn the attention to other problems in which the use of vdW-DF functional leads to a considerable improvement of the DFT calculations.
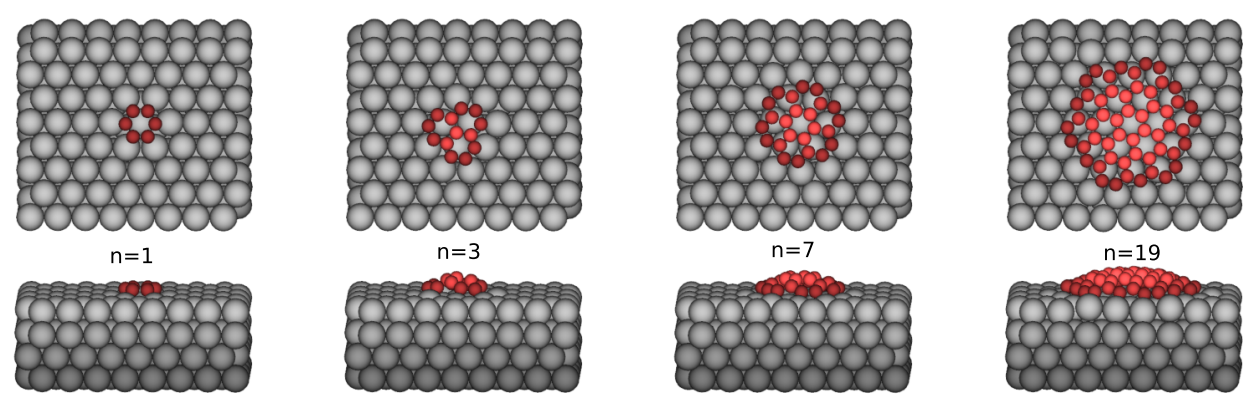

Figure 1. Geometries of graphene nanodomes on $\operatorname{Ir}(111)$ top and side view. Labeling convention: $n$ corresponds to the number of 6 -carbon atom rings contained in each dome. All geometries are shown after relaxation.

\section{Graphene nanodomes on $\operatorname{Ir}(111)$}

The growth of graphene on $\operatorname{Ir}(111)$ proceeds via the formation of small islands, called nanodomes due to their characteristic shape, as shown in figure 1. Nanodomes grow and coalesce and finally form a graphene monolayer, which can be extremely welloriented and defect-free over a large surface area. The monolayer itself is also quite complex, because the small difference of the periodicity of the components leads to a large supercell with a periodicity around $24.5 \AA$, with different relative position of $\mathrm{C}$ and Ir atoms at different points. As a consequence, the electronic properties of the graphenesubstrate bond vary over the supercell, resulting in a clearly visible moiré pattern in 
STM experiments.
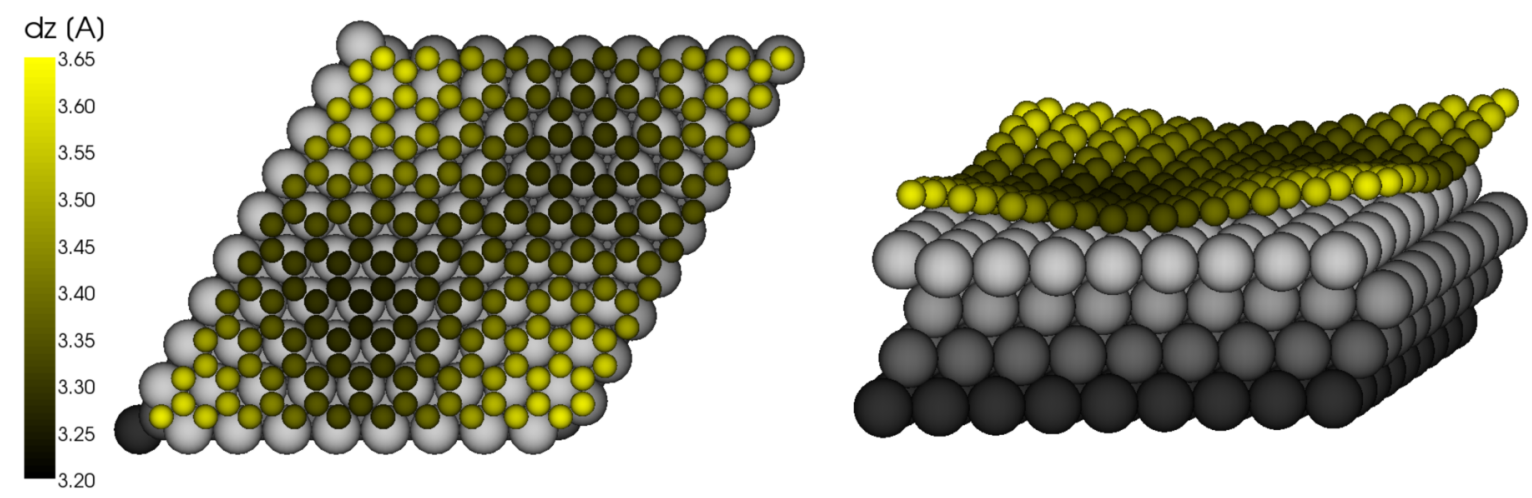

Figure 2. Moiré structure of $9 \times 9$ graphene on $10 \times 10$ iridium surface. Left: Top view, $d z$ is the distance in $\AA$ from the topmost iridium layer. Right: Side view, for clarity reasons the corrugation is magnified by a factor of five.

\subsection{Calculations}

In calculating the structures that develop during epitaxial growth of graphene on $\operatorname{Ir}(111)$ surface we closely follow the recent study of Lacovig et al. [20]. In their work they use GGA functional as implemented in the VASP code $[10,11]$ without any attempt to include dispersion forces, i.e. nonlocal correlation. In our calculations we have used the same code with its projector-augmented wave method implementation [21] and Perdew-Burke-Ernzerhof [22] type of GGA functional, with two additional features. First, we have included dispersion forces through the semi-empirical DFT-D scheme of Grimme [7] to calculate both ionic relaxations (via forces) and the total energy of the system. Second, to treat the final relaxed geometry we have performed post-processing calculation using the JuNoLo code [4] in order to get the total energy with the vdW-DF functional. In all calculations we have used $400 \mathrm{eV}$ plane wave cutoff energy, single $k$-point (except for the monolayer $9 \times 9$ over $10 \times 10$ moiré structure, where $3 \times 3 \times 1$ $k$-points were used) and convergence demand for ionic forces of $0.001 \mathrm{eV} / \AA$. Four layers of iridium were used in all calculations for the simulation of the slab, together with a vacuum region large enough to prevent spurious interactions between slab surfaces. For the full monolayer we use the large unit cell describing the observed moiré pattern, with $10 \times 10 \operatorname{Ir}(111)$ surface unit cells and $9 \times 9$ graphene unit cells, in good agreement with experiment [23]. The full geometry of the moiré structure is shown in figure 2. 


\subsection{Results and discussion}

The main results of our calculations are the adsorption energies for different structures which are shown in figure 3. From our GGA results that perfectly match the ones of Lacovig et al. [20] (except for the full monolayer) it is clear that the addition of semiempirical forces did not change the final adsorption geometry of the nanodomes, i.e. we obtain the same geometries as in the previous work. The largest difference in our results regarding the relaxed geometries is at the full monolayer coverage, i.e. the moiré structure. In our calculations the graphene layer is adsorbed at an average distance of $3.41 \AA$ and peak-to-peak corrugation of $0.4 \AA$, with a rather small adsorption energy of $50 \mathrm{meV}$ per carbon atom, indicating that in this case graphene is physisorbed. The fact that GGA functionals do not contain dispersion forces leads to problems in systems that are dominantly physisorbed which was recognized already in the original work of Lacovig et al. [20] and presented as a comment in reference 21 of their paper.

An attempt to calculate the structure of monolayer graphene on $\operatorname{Ir}(111)$ within the DFT was made earlier $[24,25,26]$. The author used both GGA and LDA, depending on the property studied, in order to avoid problems with underbinding and to get the agreement with experiment, admitting that this does not conform to the notion of working from first principles. The fact that LDA fortuitously mimics van der Waals forces by systematically overbinding, giving particularly good results in layered structures, is explained in [27]. Another test that LDA gives right numbers for wrong reasons can be easily done by shifting the graphene layer away from the Ir surface and noticing that the standard asymptotic dependence of the van der Waals layer-surface attraction energy is not reproduced. Also, by inspecting the curvature of the adsorption energy minimum and comparing it with experimental values shows that LDA does not give the correct result $[28,29]$.

The fact that our calculated adsorption energies and those of Lacovig et al. are almost identical (except for the monolayer moiré structure) shows that DFT-D does not change the original PBE geometries of nanodomes. The reason that the geometry is strongly changed at the final moiré structure can be explained as follows. Nanodomes are chemically bonded to the underlying iridium surface at their edges. As a nanodome grows larger its central part moves farther away from the surface and is more weakly bound to the surface then the edge. One can say that a dome is chemisorbed along its edge and physisorbed at its central part. As the dome grows larger the bonding mechanism shifts from dominantly chemisorbed to dominantly physisorbed, and the average adsorption energy per atom is reduced in the process. Thus, as long as the edge of the dome exists the PBE will act in some regions to produce chemisorption and adsorption will occur. However, in the case of the final moiré structure there is no edge (in a theoretical calculation, in experiment the graphene is practically always a flake with an edge) and the system is entirely physisorbed, thereby revealing the lack of dispersion forces in PBE. It is well known that PBE overbinds in chemical adsorption on surfaces [30], so in simulating the growth of nanodomes one should observe the 


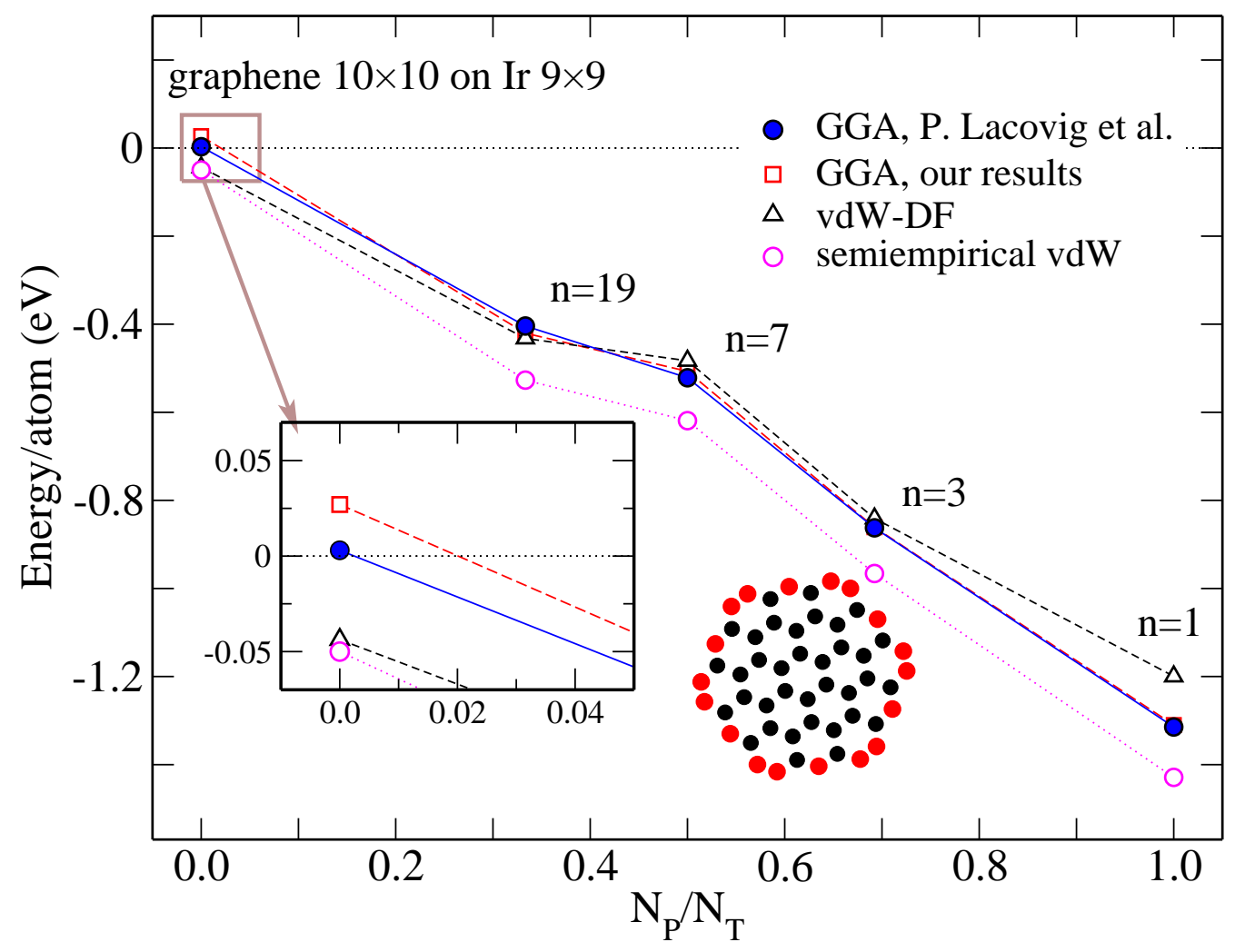

Figure 3. Adsorption energies calculated with different functionals, for several structures. Red and black circles represent $n=19$ nanodome structure defining the $N_{P} / N_{T}$ ratio as the number of carbon atoms at the edge of a dome $N_{P}$ (red circles) over the total number of atoms of the dome $N_{T}$ (red and black circles).

switching of PBE overbinding to underbinding as nanodomes grow larger. Comparing the adsorption energy values obtained with vdW-DF and PBE presented in figure 3, it becomes clear that PBE switches from overbinding to underbinding for the dome size between $n=7$ and $n=19$, where $n$ corresponds to the number of 6 -atom carbon rings contained in each dome.

By comparing the adsorption energies obtained with DFT-D the nature of this semiempirical method is revealed. DFT-D always lowers the calculated GGA total energy to compensate for the GGA's lack of nonlocal correlation, which actually gives better result than PBE alone only in the moiré structure, whereas in all other cases it incorrectly further increases the already too large adsorption energy. However, in all cases the geometry obtained by using additional DFT-D forces is very good, i.e. in the cases of domes the original PBE geometry is unchanged while in the final moiré structure the geometry is qualitatively completely different from the PBE, with no binding in PBE and with $50 \mathrm{meV}$ of binding with DFT-D, in almost perfect agreement with the vdW-DF result. The conclusion that DFT-D geometry for the moiré pattern is good is derived by comparison with the experimental data [31]. 
The above calculations show that a feasible strategy to obtain correct adsorption energies is to use additional semiempirical forces for geometric relaxation (that come at no additional computational cost) together with the GGA calculation, and to perform vdW-DF calculation for the final geometry in order to get accurate adsorption energy. This postprocessing usage of $\mathrm{vdW}-\mathrm{DF}$ is practically equivalent to a selfconsistent implementation (for a given structure) that was presented in [32]. The same strategy was successfully employed earlier [33], and recently it was demonstrated in a direct comparison with experiment [34] that the geometries obtained with DFT-D are very accurate, but the adsorption energies are not. We find a simple explanation for this as follows - DFT-D always lowers the calculated GGA total energy (unlike vdW-DF), and adds only attractive forces between the parts of the system, tending to bring them closer together. In cases in which the parts of the system are in pure van der Waals interaction they will be correctly brought closer, and DFT-D is best tailored for description of this situation, including the prediction of energy as well. On the other hand, if some parts of the system are chemically bonded, which is more or less correctly captured within GGA, the DFT-D will not change the geometry further because the chemical bond is characterized by a very rapid onset of Pauli repulsion when the atoms are brought closer than their equilibrium distance. The geometry remains practically unchanged by adding DFT-D forces due to the small binding energy that DFT-D brings in.

In the present example of the epitaxial growth of graphene on $\operatorname{Ir}(111)$ surface, in which the PBE crosses over from overbinding to underbinding following the growth of the domes, it is shown how the PBE completely fails qualitatively only at the monolayer coverage, i.e. at the moiré structure. In that case very good results are also obtained with the aid of semiempirical methods such as DFT-D.

\subsection{Definition of the binding energy densities}

The vdW-DF calculations with JuNoLo code are performed in the post-processing procedure, in which all energies are calculated using the charge density obtained from a selfconsistent PBE calculation. The PBE correlation energy, $E_{\mathrm{c}}^{\mathrm{PBE}}$, is replaced by the sum of a local correlation energy in the local density approximation, $E_{\mathrm{c}}^{\mathrm{LDA}}$, and the nonlocal contribution, $E_{\mathrm{c}}^{\mathrm{NL}}$,

$$
E_{\text {tot }}^{\mathrm{NL}}=E_{\mathrm{tot}}^{\mathrm{DFT}}-E_{\mathrm{c}}^{\mathrm{PBE}}+\left(E_{\mathrm{c}}^{\mathrm{LDA}}+E_{\mathrm{c}}^{\mathrm{NL}}\right)
$$

which is given by a double-space integral:

$$
E_{\mathrm{c}}^{\mathrm{NL}}=\frac{1}{2} \iint d^{3} \mathbf{r} d^{3} \mathbf{r}^{\prime} n(\mathbf{r}) \phi(\mathbf{r}, \mathbf{r}) n\left(\mathbf{r}^{\prime}\right)
$$

Our analysis resorts to the density weighted correlation energy-density $e_{\mathrm{c}}(\mathbf{r})$

$$
E_{\mathrm{c}}=\int d^{3} \mathbf{r} n(\mathbf{r}) \epsilon_{\mathrm{c}}(\mathbf{r})=\int d^{3} \mathbf{r} e_{\mathrm{c}}(\mathbf{r})
$$

We are particularly interested in the differences between the correlation binding-energy densities obtained with the PBE and the nonlocal vdW-DF functional. Binding energy 
is the difference between the energy of a combined (compound) system consisting of a structurally fully relaxed object on the substrate, and the energy of disconnected parts (object and substrate) with the atoms at exactly the same positions as obtained in the original calculation. The correlation binding-energy (BE) density is then given by:

$$
\left.\left.e_{\mathrm{c}}^{\mathrm{BE}}(\mathbf{r})=e_{\mathrm{c}}^{\mathrm{system}}(\mathbf{r})-\right] e_{\mathrm{c}}^{\mathrm{part} 1}(\mathbf{r})+e_{\mathrm{c}}^{\mathrm{part} 2}(\mathbf{r})\right] .
$$
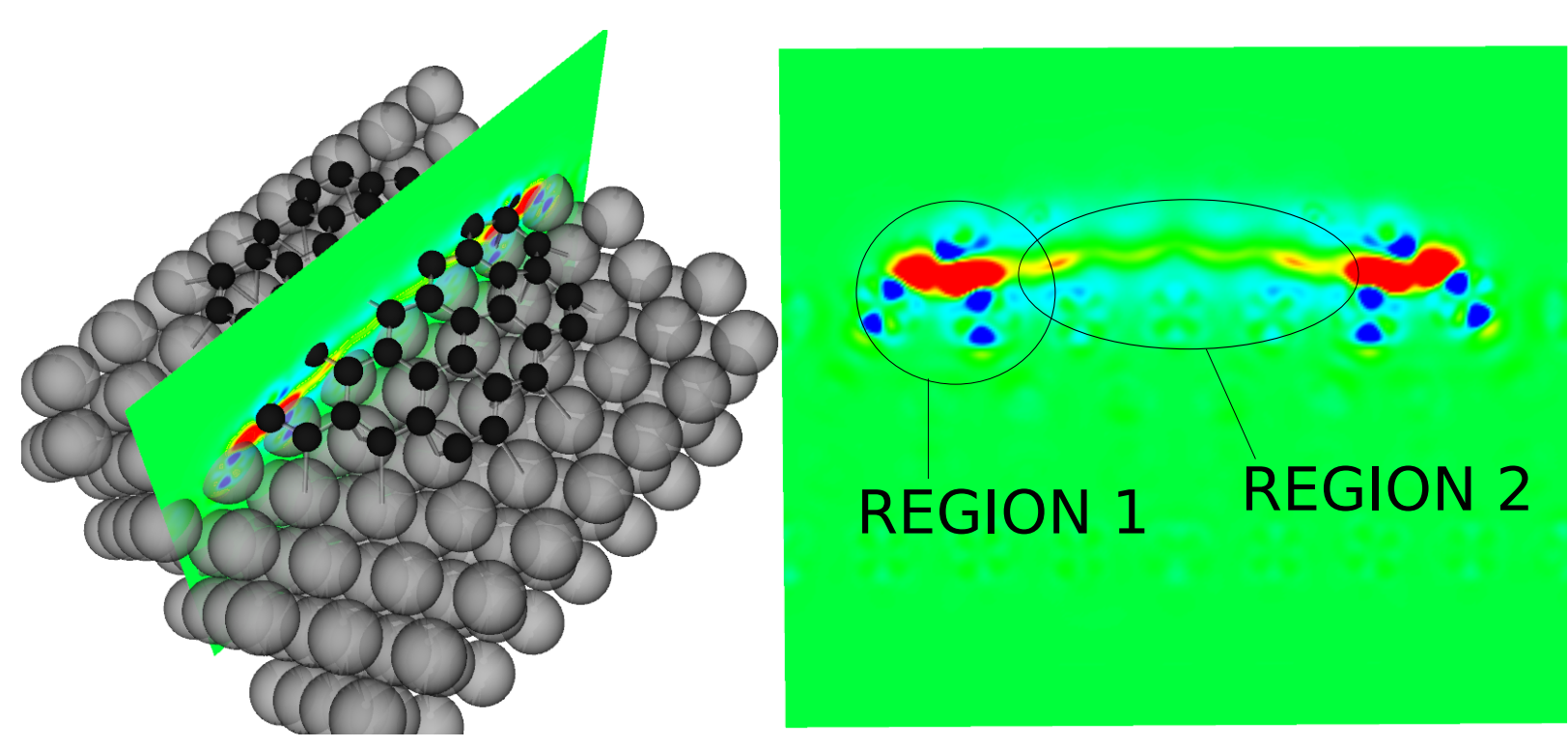

Figure 4. PBE correlation overbinding (red) and PBE underbinding (blue) compared to vdW-DF shown in the cross-section plane (green color shows zero value). Region 1 is the region at the edge of the dome showing dominantly chemical bonding, while region 2 is at the center of the dome where vdW binding dominates. For better view see animation $[35,36]$.

In a calculation with PBE (or any other semilocal correlation functional) it is obvious that the correlation energy can be assigned to a particular point in space $\mathbf{r}$, because of its definition. For the nonlocal contribution given by a double-space integral of (2) we rewrite the expression as:

$$
\begin{aligned}
E_{\mathrm{c}}^{\mathrm{NL}} & =\frac{1}{2} \iint d^{3} \mathbf{r} d^{3} \mathbf{r}^{\prime} n(\mathbf{r}) \phi\left(\mathbf{r}, \mathbf{r}^{\prime}\right) n\left(\mathbf{r}^{\prime}\right) \\
& =\int d^{3} \mathbf{r} n(\mathbf{r}) \epsilon_{\mathrm{c}}^{\mathrm{NL}}(\mathbf{r})=\int d^{3} \mathbf{r} e_{\mathrm{c}}^{\mathrm{NL}}(\mathbf{r}) .
\end{aligned}
$$

Thereby we obtain the quantity $e_{\mathrm{c}}^{\mathrm{NL}}(\mathbf{r})$ that is defined at each point in space and we designate it the density weighted nonlocal correlation energy density. Once we have $e_{\mathrm{c}}^{\mathrm{BE}(\mathrm{PBE})}(\mathbf{r})$ and $e_{\mathrm{c}}^{\mathrm{BE}(\mathrm{vdW}-\mathrm{DF})}(\mathbf{r})$ and compute their difference at each space point

$$
\Delta e_{\mathrm{c}}^{\mathrm{BE}}(\mathbf{r})=\left(e_{\mathrm{c}}^{\mathrm{BE}(\mathrm{LDA})}(\mathbf{r})+e_{\mathrm{c}}^{\mathrm{BE}(\mathrm{NL})}(\mathbf{r})\right)-e_{\mathrm{c}}^{\mathrm{BE}(\mathrm{PBE})}(\mathbf{r})
$$

we obtain the correlation binding-energy difference. The same quantity was defined in [37]. 


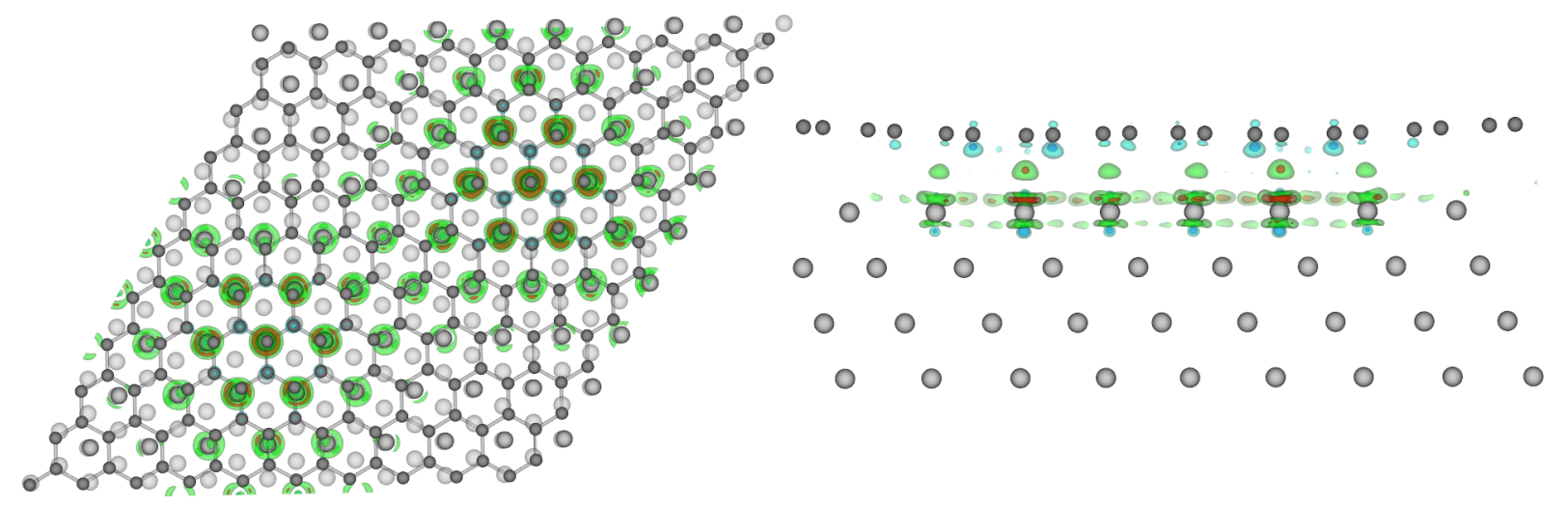

Figure 5. Charge transfer for the moiré structure top (left) and side (right) view. Red color shows charge accumulation and blue colors show charge depletion. For better view see animation [38].

\subsection{Over- and underbinding in PBE}

Since we consider the vdW-DF calculation to be more accurate than the PBE one, we determine the regions of space in which $\Delta e_{\mathrm{c}}^{\mathrm{BE}}(\mathbf{r})>0(<0)$ and designate them as PBE-overbinding (PBE-underbinding). Those regions are shown in the cross section in figure 4.

To visualize these regions in three dimensions the animation is much more appropriate $[35,36]$. In animation [35] one can see that PBE is overbinding in the regions where the chemical bonds are present and that it is underbinding in the regions closer to atoms at the edge. This corresponds to region 1 marked in figure 4 . There is also an underbinding contribution in the middle of the dome (region 2 in figure 4), but it is not visible in the animation due to its small value. Therefore, we have made an additional animation [36], which shows isosurfaces with slightly lower values. One should keep in mind that only the integration of this quantity over the entire space gives the total underbinding energy so that large regions of low density can add up to produce significant values of adsorption energy. This is actually the principle how the nonlocal interaction starts to dominate the binding as the domes grow larger. The regions of PBE overbinding grow linearly with the dome circumference because they are located only at the dome's edge (region 1) while the regions of PBE underbinding (region 2) grow with the dome's surface. In this way it is clear how the adsorption energy calculated with PBE and shown in figure 3 becomes too large for small domes and too small for larger ones.

\subsection{Nonlocal correlation binding energy density}

Here we present the quantity $e_{\mathrm{c}}^{\mathrm{BE}(\mathrm{NL})}(\mathbf{r})$, defined by $(4,5)$ and calculated for a $10 \times 10$ graphene on $9 \times 9 \operatorname{Ir}(111)$ moiré structure, because this contribution is responsible for almost all of the binding energy of the whole system. The system is almost perfectly 

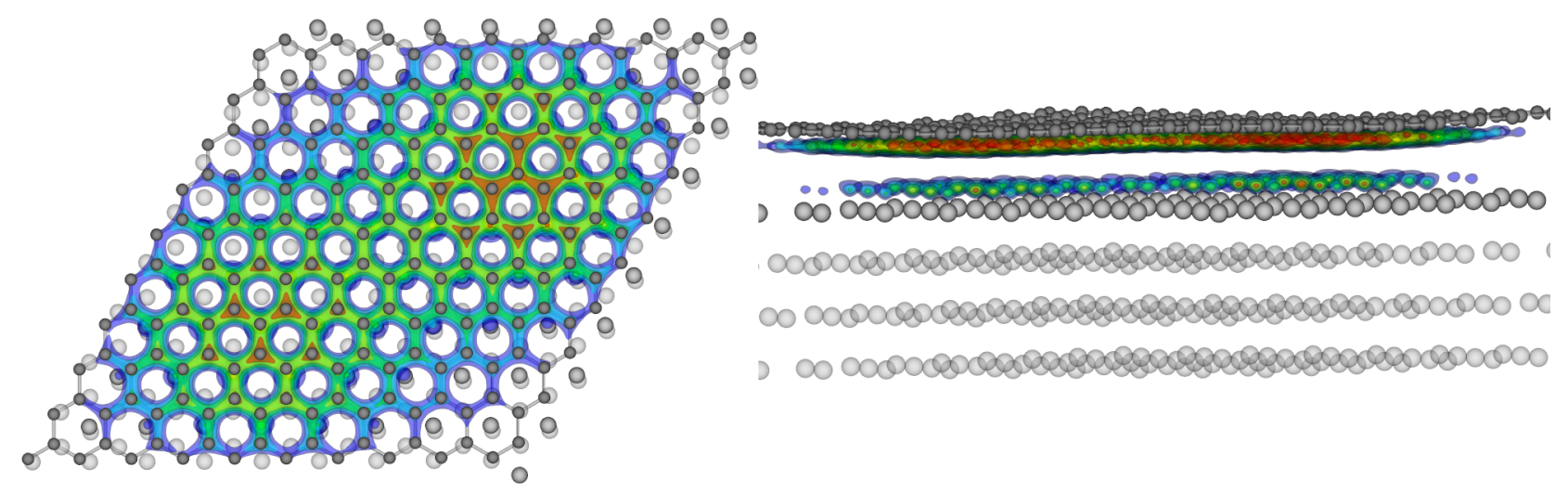

Figure 6. Nonlocal correlation binding energy density for moiré structure. Top (left) and side (right) view. Red color is the highest value and blue is the lowest value. For better view see animation [39].

physisorbed with a remnant charge transfer from graphene to Ir substrate of around 0.02 electrons per $\mathrm{C}$ atom. The charge transfer for this structure is shown in figure 5 and as animation in [38].

The nonlocal binding energy density is shown in figure 6 and in the animation in [39]. This demonstrates that the interaction is nonlocal on the length scale of a relatively large iridium-graphene distance since one of its components is located just below the graphene sheet, whereas the other is just above the iridium surface.

\section{The CO Puzzle}

The fact that PBE overbinds is sometimes seen as a quantitative problem, but when comparing systems of similar structure and similar adsorption energies this quantitative overbinding error can turn into qualitatively wrong prediction. A well known example of this is the $\mathrm{CO}$ adsorption puzzle [40], that was eventually resolved by introducing the nonlocal correlation [41, 42] and reconsidered recently [43].

In $\mathrm{CO}$ adsorption on (111) surfaces of $\mathrm{Pt}, \mathrm{Ru}, \mathrm{Cu}$ and some other metals the DFT calculations with semilocal functionals predict that the adsorption at FCC hollow site is preferred in comparison to atop site. On the other hand, in experiments adsorption at the FCC sites has never been experimentally observed in these systems, only at atop sites. At larger coverages some adsorption at bridge sites is also detected. This problem can not be remedied by the use of hybrid exchange functionals [44], which shows that a large part of the problem is not in the exchange part of the functional. Only the introduction of nonlocal correlations resolves this puzzle, through exactly the same mechanism that reduces the PBE overbinding in the graphene nanodomes. The other option to account for nonlocal correlation is to use the RPA approach which is computationally much more expensive than the vdW-DF and difficult to implement selfconsistently. In principle it should do exactly the same job as the vdW-DF so that we agree with the conclusions of [16] that the ab-initio community should use 
nonlocal functional(s) such as vdW-DF rather than the RPA approach and hopefully develop new nonlocal functionals. Also, RPA is not in the true spirit of the density functional theory as it is not based exclusively on the density. Resolution of the $\mathrm{CO}$ puzzle can be described simply by starting from the overbinding of PBE functional in chemical bonds. Adsorption of $\mathrm{CO}$ on the (111) surface of metals into sites with different coordination, such as top and FCC, site results in different numbers of regions in which the charge transfer between $\mathrm{CO}$ molecule and metallic surface occurs (formation of chemical bonds). In the case of top site adsorption there is only one such region while in the case of FCC there are three such regions, separated in space. The strength of the single bond in the case of top site adsorption is larger than each bond in the FCC case. However, the PBE overbinding occurs independently in all three regions of charge transfer in the FCC adsorption and only in one region in the case of top site adsorption. This effect incorrectly pushes the adsorption preference towards the sites with higher coordination and when adsorption energies are similar in the two cases it may even result in a seemingly qualitatively wrong prediction. The length scales (1$2 \AA$ ) at which nonlocal correlation becomes important are much smaller than the typical vdW distances, they are of the size of the chemical bond - but still the PBE or any other GGA functional can not capture these features correctly. For a detailed analysis see $[42,41]$.

\section{The role of the exchange functional}

In the original formulation of the vdW-DF functional [1], the nonlocal correlation (2) was used together with the semilocal revPBE functional for the exchange part, on the grounds that that the more standard PBE exchange predicts spurious exchange-only binding of rare gas dimers. The resulting interaction energies for $\mathrm{Ar}$ and $\mathrm{Kr}$ dimers agreed well with experiment, but interatomic distances were somewhat too large. Later studies showed that revPBE exchange is often too repulsive in the binding region. In calculations of systems with strong covalent or hydrogen bonding [37, 42], it was found that the PBE exchange gives better results than the revPBE. It was soon realised that the question of the most appropriate GGA exchange (or possibly a hybrid exchange) in conjunction with the nonlocal correlation functional is an open question, vital for further development of the vdW-DF and its application to a wide range of systems. This is not surprising considering that within the domain of GGA DFT, in particular in chemical applications, a number of different GGA and hybrid exchange functionals have been proposed and are currently used. The choice depends on the corresponding GGA correlation, and to some extent on the system being considered. (The latter, however, is against the spirit of universality and transferability of DFT).

In the last few years a number of exchange functionals for vdW-DF have been considered. In [19] the exchange functional C09 was proposed and evaluated with the benchmark S22 database set of weakly interacting dimers. The PW86 exchange was identified as a promising possibility and a refitted version rPW86 was used in the revised 
vdW-DF2 functional, in which the nonlocal correlation part was also slightly modified. Klimeš et al. [17] evaluated a number of new and published exchange functionals on the S22 set and water clusters, and identified those that deliver better than chemical accuracy. In a later paper [12] the original vdW-DF and several of its offsprings were tested on traditional "hard" matter, such as metals, ionic compounds, and insulators. Although these studies do not yet indicate clearly the best choices, they should be useful in the further development of the method.

A particularly challenging system for DFT simulations is graphene adsorbed on $\mathrm{Ni}(111)$ surface. At first sight, the system appears simple as the lattice constants of the two structures match almost perfectly and a simple $1 \times 1$ unit cell can be used, unlike e.g. graphene on $\operatorname{Ir}(111)$ where a large supercell had to be used in order to realistically simulate the moiré superstructure, cf. figure 2. First DFT calculations using the LDA functional [45] gave the graphene-substrate distance of $2.05 \AA$, which implies a strong adsorption, in agreement with ARPES experiments which show that the Dirac cones of the graphene electronic bands are completely destroyed. A subsequent study [46] showed that the standard GGA and vdW-DF do not perform well in this case. The GGA functionals predict virtually no adsorption, which is not surprising, but the nonlocal vdW-DF with revPBE exchange only partially corrects this, giving only a weak vdW physisorption at $3.50 \AA$ above the substrate and the Dirac cones remain almost intact, at variance with experiment.

There is a fundamental difference between graphene on $\mathrm{Ni}(111)$ and the systems considered until now in that the nickel substrate is magnetic. This immediately suggests that the choice of the exchange functional in DFT calculations may be critical. In fact, further research showed [47] that while the original vdW-DF and vdW-DF2 perform poorly, the respective nonlocal correlation functionals combined with C09 exchange give a very strong adsorption around the expected graphene-substrate distance of just over $2 \AA$. Mittendorfer et al. [48] examined the problem using vdW-DF with several other exchange functionals, and compared the results with a full RPA calculation. They found that the interaction energy curve has two minima, one corresponding to physisorption and a second one closer to the surface, at $2.17 \AA$, with the energy minimum slightly lower but still much smaller than expected for typical chemisorption. The RPA results could be very well reproduced by DFT calculation with optB88 exchange, which gave the binding energy at the two minima very close to the RPA values.

These examples show that DFT calculations with nonlocal correlation functionals initiated by Dion et al. [1], can be applied on a large number of materials and can cover interactions of various nature and strength. The search for the most appropriate exchange functional is probably the most important issue in order to make the vdW-DF class of functionals at least as good as the modern GGA functionals, extending the applicability of DFT to cases where GGA does not perform well. 


\section{Conclusions}

In conclusion, we have shown on the example of the growth of graphene nanodomes on the $\operatorname{Ir}(111)$ surface that PBE calculations encounter problems. For small domes PBE yields too large adsorption energies while for larger ones the adsorption energy is too small, ultimately failing at the moiré structure that, according to the PBE, is not even binding. It may seem that the application of semi-empirical scheme such as DFT-D remedies this by restoring the binding, but it is not a general improvement. Besides being semi-empirical and non electronic-based (i.e. it is based on ionic positions only rather than on the electronic density) we have shown that the energies derived from it are unreliable even though the geometries obtained by using DFT-D forces are very good. There are also some other issues associated with DFT-D, such as the omission of image plane as discussed in [49]. DFT-D is quite handy for sparse matter systems but does not represent a systematic improvement in DFT. On the other hand, the introduction of a nonlocal density functional, such as vdW-DF, represents a very important general improvement in DFT calculations, and this is not limited only to sparse matter systems. By taking the example of graphene on $\operatorname{Ir}(111)$ from this work, and combining it with the results of [42] in which the CO puzzle was solved, we have shown how the vdWDF resolves the major problems of DFT implementation in two completely different types of systems, viz. the chemisorbed and the physisorbed ones, and how it seamlessly interpolates between these two extremes. The best examples of systems that lie between chemisorption and physisorption are those of organic molecules on metallic surfaces for which physisorption and chemisorption are blended together so that a seamless functional is essential for a proper DFT treatment. Such systems have already been studied [33] and revisited [43]. It is worth mentioning that a pioneering work in the area of transport calculations indicates that the lack of nonlocality in the functionals causes even bigger problems, leading to completely wrong qualitative predictions [50]. In summary, by revealing the full meaning of the seamless functional for the vdW-DF, we have shown that nonlocal functionals prove indispensable in the DFT calculations. Their implementation should relieve the users from the concerns of which functional to use for which system, thereby justifying DFT's ab initio attribute and strengthening its predictive power.

\section{Acknowledgments}

The computations were performed on JUROPA and JUGENE supercomputers at the Jülich Supercomputing Center, Forschungszentrum Jülich, Germany. P. L. acknowledges financial support from the Alexander von Humboldt Foundation. R. B. and B. G. acknowledge the support by the Ministry of Science and Technology of the Republic of Croatia under contract nos 098-0352828-2863 and 035-0352828-2839, respectively. 


\section{References}

[1] M. Dion, H. Rydberg, E. Schröder, D. C. Langreth, and B. I. Lundqvist, Physical Review Letters 92, 246401 (2004).

[2] M. Dion, H. Rydberg, E. Schröder, D. C. Langreth, and B. I. Lundqvist, Physical Review Letters 95, 109902(E) (2005).

[3] P. Hohenberg and W. Kohn, Physical Review 136, B864 (1964).

[4] P. Lazić et al., Computer Physics Communications 181, 371 (2010).

[5] G. Román-Pérez and J. M. Soler, Physical Review Letters 103, 096102 (2009).

[6] S. Grimme, J. Comput. Chem. 25, 1463 (2004).

[7] S. Grimme, J. Comput. Chem. 27, 1787 (2006).

[8] S. Grimme, J. Antony, S. Ehrlich, and H. Krieg, The Journal of Chemical Physics 132, 154104 (2010).

[9] J. Enkovaara et al., Journal of Physics: Condensed Matter 22, 253202 (2010).

[10] G. Kresse and J. Furthmüller, Physical Review B 54, 11169 (1996).

[11] G. Kresse and J. Joubert, Physical Review B 59, 1758 (1999).

[12] J. Klimeš, D. R. Bowler, and A. Michaelides, Physical Review B 83, 195131 (2011).

[13] K. Berland, Ø. Borck, and P. Hyldgaard, 1007.3305 (2010).

[14] D. C. Langreth et al., Journal of Physics: Condensed Matter 21, 084203 (2009).

[15] J. Harl and G. Kresse, Physical Review B 77, 045136 (2008).

[16] J. Harl and G. Kresse, Physical Review Letters 103, 056401 (2009).

[17] J. Klimeš, D. R. Bowler, and A. Michaelides, Journal of Physics: Condensed Matter 22, 022201 (2010).

[18] K. Lee, E. D. Murray, L. Kong, B. I. Lundqvist, and D. C. Langreth, Physical Review B 82, 081101 (2010).

[19] V. R. Cooper, Physical Review B 81, 161104 (2010).

[20] P. Lacovig et al., Physical Review Letters 103, 166101 (2009).

[21] P. E. Blöchl, Physical Review B 50, 17953 (1994).

[22] J. P. Perdew, K. Burke, and M. Ernzerhof, Physical Review Letters 77, 3865 (1996).

[23] I. Pletikosić et al., Physical Review Letters 102, 056808 (2009).

[24] A. T. N'Diaye, S. Bleikamp, P. J. Feibelman, and T. Michely, Physical Review Letters 97, 215501 (2006).

[25] A. T. N'Diaye, S. Bleikamp, P. J. Feibelman, and T. Michely, Physical Review Letters 101, 219904 (erratum) (2008).

[26] P. J. Feibelman, Physical Review B 77, 165419 (2008).

[27] E. Londero and E. Schröder, Physical Review B 82, 054116 (2010).

[28] P. Lazić, Ž. Crljen, R. Brako, and B. Gumhalter, Physical Review B 72, 245407 (2005).

[29] P. Lazić, R. Brako, and B. Gumhalter, Journal of Physics: Condensed Matter 19, 305004 (2007).

[30] B. Hammer, L. B. Hansen, and J. K. Nørskov, Physical Review B 59, 7413 (1999).

[31] C. Busse et al., Physical Review Letters 107, 036101 (2011).

[32] T. Thonhauser et al., Physical Review B 76, 125112 (2007).

[33] N. Atodiresei, V. Caciuc, P. Lazić, and S. Blügel, Physical Review Letters 102, 136809 (2009).

[34] G. Mercurio et al., Physical Review Letters 104, 036102 (2010).

[35] http://www.youtube.com/watch?v=WoOabfYxffQ .

[36] http://www.youtube.com/watch?v=209KV3o63-E .

[37] A. Gulans, M. J. Puska, and R. M. Nieminen, Physical Review B 79, 201105 (2009).

[38] http://www.youtube.com/watch?v=mxTKhb0wgq4 .

[39] http://www.youtube.com/watch?v=PEYcBD7UlhE .

[40] P. J. Feibelman et al., The Journal of Physical Chemistry B 105, 4018 (2001).

[41] P. Lazić et al., 0810.2269 (2008).

[42] P. Lazić et al., Physical Review B 81, 045401 (2010). 
[43] L. Schimka et al., Nat Mater 9, 741 (2010).

[44] A. Stroppa and G. Kresse, New Journal of Physics 10, 063020 (2008).

[45] P. A. Khomyakov et al., Physical Review B 79, 195425 (2009).

[46] M. Vanin et al., Physical Review B 81, 081408 (2010).

[47] I. Hamada and M. Otani, Physical Review B 82, 153412 (2010).

[48] F. Mittendorfer et al., Physical Review B 84, 201401 (2011).

[49] K. Berland and P. Hyldgaard, The Journal of Chemical Physics 132, 134705 (2010).

[50] J. S. Evans, O. A. Vydrov, and T. V. Voorhis, The Journal of Chemical Physics 131, 034106 (2009). 